\title{
Current management of vesicoureteral reflux in pediatric patients: a review
}

This article was published in the following Dove Press journal:

Pediatric Health, Medicine and Therapeutics

14 February 2013

Number of times this article has been viewed

\author{
Aviva E Weinberg \\ Michael H Hsieh \\ Department of Urology, Stanford \\ University School of Medicine, \\ Stanford, CA, USA
}

\begin{abstract}
Vesicoureteral reflux (VUR) is both a functional and anatomic pediatric disorder arising from incompetence of the ureterovesical junction. Some children with VUR remain entirely asymptomatic, while others may develop pyelonephritis, renal scarring, and potentially progressive renal insufficiency. There are still many areas of VUR diagnosis, management, and timing of intervention that remain controversial today. This review attempts to synthesize the best available current evidence from the literature dealing with VUR screening, diagnosis, and treatment in order to provide the practitioner with an organized and evidence-based approach to the disease.
\end{abstract}

Keywords: vesicoureteral, children, treatment, antibiotics, prophylaxis, reimplantation

\section{Introduction}

Vesicoureteral reflux (VUR), the abnormal retrograde flow of urine into the upper urinary tract, is both an anatomical and functional pediatric disorder. In many cases, VUR remains entirely asymptomatic, while reflux in the presence of bacteriuria puts the child at risk for pyelonephritis, renal scarring, renal dysplasia, and potentially progressive renal insufficiency. The appropriate evaluation and management algorithm for VUR has remained a complex and contentious issue, as screening, diagnosis, and treatment guidelines continue to evolve alongside a continually expanding body of VUR literature. There are still many areas of VUR diagnosis (top-down versus bottom-up approaches), management (observation, medical, endoscopic, surgical) and timing of intervention that remain controversial today. This review attempts to synthesize the best available current evidence from the literature dealing with VUR screening, diagnosis, and treatment in order to provide the practitioner an organized and evidence-based approach to VUR management.

\section{Epidemiology and etiology}

VUR is a very common condition among children, with prevalence rates that vary based on patient age, sex, race, family history, and associated urologic anomalies. It may be an isolated diagnosis, or found in combination with a variety of urologic conditions, such as posterior urethral valves, duplicated collecting system, multicystic dysplastic kidney, prune-belly syndrome, bladder exstrophy, lower urinary tract dysfunction, and ureteropelvic junction obstruction. Since VUR can be asymptomatic and lower grades often spontaneously resolve, it is challenging to calculate a true prevalence rate. Older estimates of VUR prevalence among asymptomatic children have cited a rate of
Correspondence: Michael H Hsieh Department of Urology, Room S287, Stanford University School of Medicine, 300 Pasteur Drive, Stanford, CA 94305-5। 18, USA

Tel +l 6507239779

Fax +l 6507252502

Email mhhsieh@stanford.edu article which permits unrestricted noncommercial use, provided the original work is properly cited. 
$0.4 \%-1.8 \% .^{1}$ More recently, Sargent completed a review of studies of children undergoing voiding cystourethrography (VCUG) for various indications, estimating the prevalence of reflux in normal children without any urologic history at $9 \%$, and as high as $30 \%$ for those presenting with a history of a urinary tract infection (UTI). ${ }^{2}$

Irrespective of the exact prevalence of VUR in children as a whole, there are distinct sex and race differences. Early in life, boys are more likely to have VUR; however, the majority of persistent reflux in older children is found in girls. A retrospective review of 15,504 children by Chand et al reported a female-to-male ratio of reflux of $2: 1 .^{3}$ Additionally, they found that white children were three times more likely to be diagnosed with VUR than black children. Accordingly, VUR certainly has a genetic component, but the precise mode of inheritance remains unknown. The reported prevalence of VUR in screened populations is $27 \%$ in siblings and $36 \%$ in offspring. ${ }^{4}$ Currently, researchers hypothesize that VUR is inherited in an autosomal dominant fashion with variable penetrance.

VUR may be classified as primary reflux or secondary reflux, depending on the underlying etiology. Primary reflux is a congenital anatomic disorder due to a laterally displaced, incompetent ureterovesical junction that is lacking sufficient submucosal tunnel length. Secondary reflux is a functional disorder that occurs as the result of predisposing lower urinary tract dysfunction. Contributing etiologies include various causes of neurogenic bladder such as spina bifida or myelomeningocele, obstructive pathologies such as posterior urethral valves, or underlying voiding dysfunction with highpressure voiding.

VUR is typically graded using the grading system formulated by the International Reflux Committee, which classifies VUR into grades I-V, based on the degree of pelvic, calyceal and ureteral dilatation seen on $\mathrm{VCUG}^{5}$ (Figure 1). Historically, this grading system has been used to stratify patients by predicted resolution rates and proposed treatment algorithms. Estrada et al developed a clinical nomogram for predicting VUR spontaneous-resolution rates using a combination of age at presentation, sex, VUR grade, laterality, mode of clinical presentation, and ureteral anatomy. ${ }^{6}$ These predictive models, once validated, can be used to aid practitioners in counseling families and developing individualized management decisions.

The diagnostic evaluation of VUR consists of a detailed patient and family medical history, screening for lower urinary tract dysfunction, urinalysis, and imaging studies. Radiographic findings are key to guiding therapeutic management. Renal bladder ultrasonography (RBUS), VCUG, and dimercaptosuccinic acid (DMSA) scintigraphy are the customary imaging studies currently employed. The gold-standard imaging study for the diagnosis of VUR is VCUG. It provides the most detailed anatomy and allows for accurate VUR grading (Figures 2-6). Radionuclide cystograms have also been utilized due to their lower radiation exposure and higher sensitivity; however, the anatomical depiction is less precise and does not allow for initial, highresolution grading of disease severity. ${ }^{7}$ DMSA scans can be

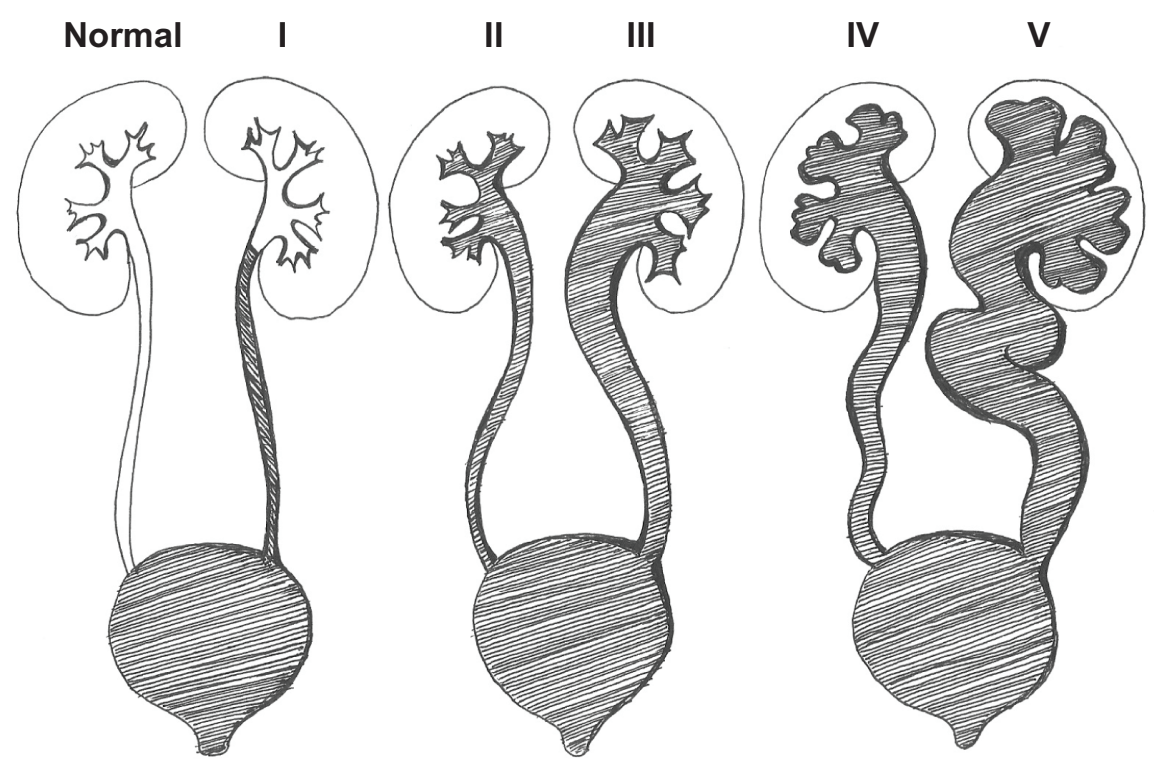

Figure I International classification of vesicoureteral reflux.

Note: Drawing by AE Weinberg. Based on International Reflux Committee. Medical versus surgical treatment of primary vesicoureteral reflux. Pediatrics. 1981;67:392.69 


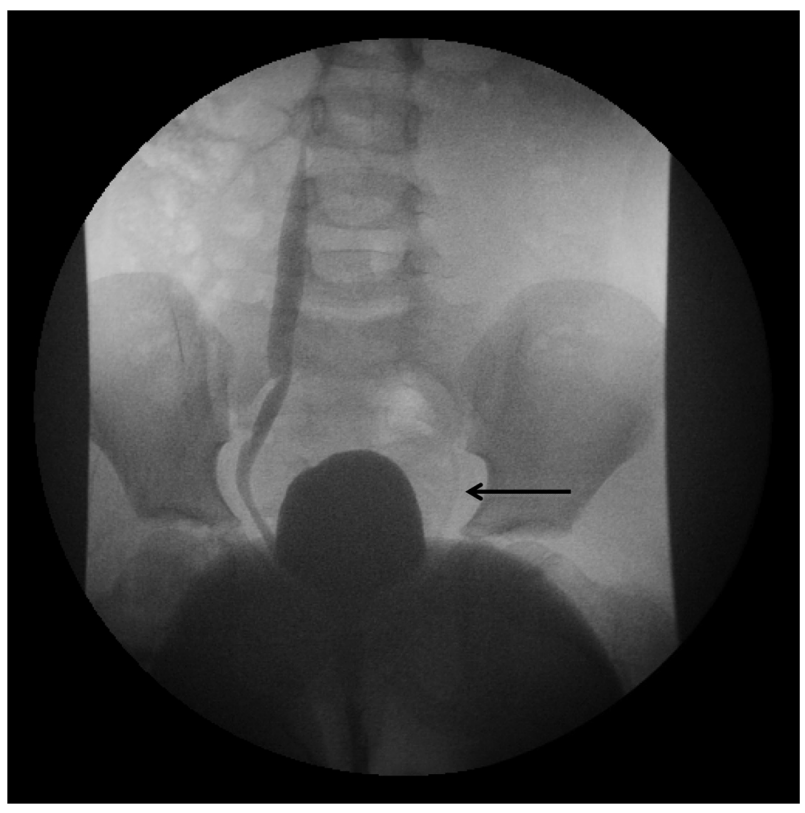

Figure 2 Voiding cystourethrogram demonstrating grade III vesicoureteral reflux on the right and grade I reflux on the left (arrow).

used to diagnose a suspected episode of acute pyelonephritis and later to assess differential renal function and the degree of renal cortical scarring ${ }^{8}$ (Figure 7). Urodynamic studies are only indicated in children suspected to have secondary reflux. Emerging imaging modalities such as voiding urosonography, magnetic resonance VCUG, and noninvasive thermal bladder and kidney imaging are currently being tested and hold hope for future clinical application. ${ }^{9}$

\section{Diagnosis of VUR after first febrile UTI}

The guidelines for the diagnostic evaluation of a child presenting with a first febrile UTI have undergone several revisions in parallel with the shifting evidence regarding the biology of VUR. Previous American Academy of Pediatrics guidelines recommended RBUS and VCUG for any child between 2 and 24 months of age who presented with their first febrile UTI. ${ }^{10}$ However, in 2011, these guidelines were revised. Now, only RBUS is recommended after the initial febrile UTI. VCUG is indicated if the RBUS reveals hydronephrosis, scarring, or any other findings that would potentially indicate either highgrade VUR or obstructive uropathy, and in cases of recurrent febrile UTI. ${ }^{11}$ These changes were generated by recent literature suggesting that the proportion of infants with high-grade VUR among infants with febrile UTIs is lower than originally appreciated, that antimicrobial prophylaxis in the setting of VUR is likely of less benefit than once thought, and finally that VUR is not an absolute requirement for the development

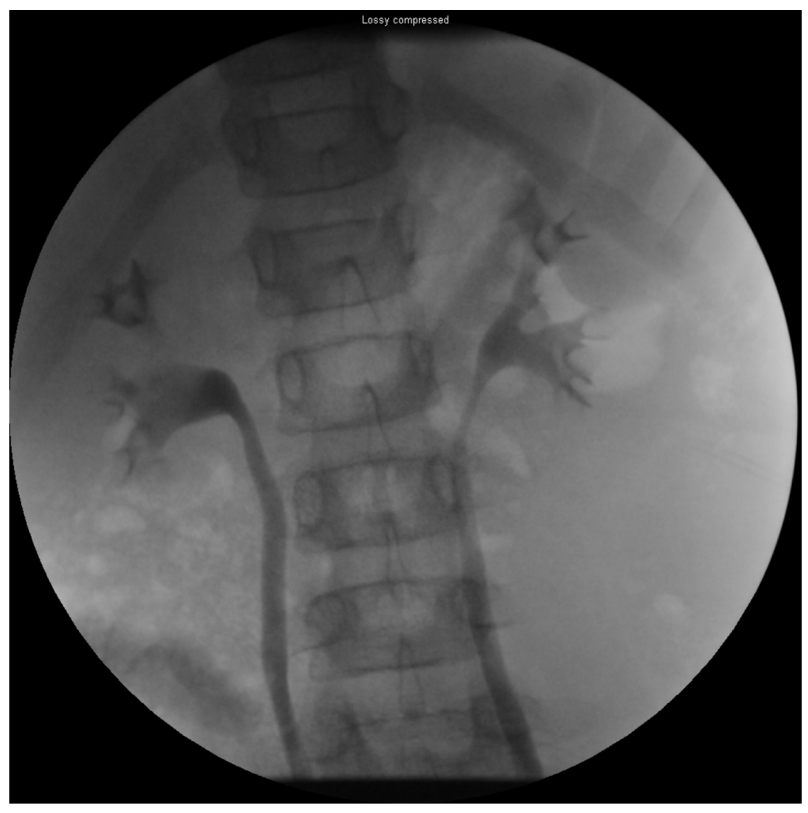

Figure 3 Voiding cystourethrogram demonstrating bilateral grade II vesicoureteral reflux.

of pyelonephritis. ${ }^{12,13}$ In contrast to the recent American Academy of Pediatrics guidelines, the European Association of Urology (EAU) continues to recommend VCUG after the first febrile UTI in children aged 0-2 years. ${ }^{14}$ They additionally recommend a DMSA scan if VUR is diagnosed on initial VCUG. The American Urological Association (AUA) guidelines recommend DMSA scanning only in the presence of an abnormal RBUS, in the setting of breakthrough UTIs, in those with high-grade VUR (grade III-V) or for elevated serum creatinine. ${ }^{15}$

Alternatively, a "top-down approach" is suggested, in which a DMSA scan is performed after the initial febrile UTI, and is followed by VCUG only if pyelonephritis or renal scarring is identified. The recent National Institute for Health and Clinical Excellence guidelines advocate initial renal ultrasound or DMSA scan (4-6 months postinfection in order to assess renal scarring rather than pyelonephritis) in children with their first febrile UTI, with VCUG reserved for those children who have abnormal DMSA results. ${ }^{16}$ Herz et al also have tested the "top-down approach;" they performed an early post-UTI DMSA scan (to detect pyelonephritis) followed by another DMSA scan $\sim 6$ months later (to detect renal scarring) if the first scan showed pyelonephritis. This approach featured 96\% sensitivity, $72 \%$ specificity, $83 \%$ positive predictive value, and $92 \%$ negative predictive value for clinically significant VUR. Their data demonstrates that a normal post-UTI DMSA scan will help avoid unnecessary VCUGs in $35 \%-60 \%$ of screened children. ${ }^{17}$ 


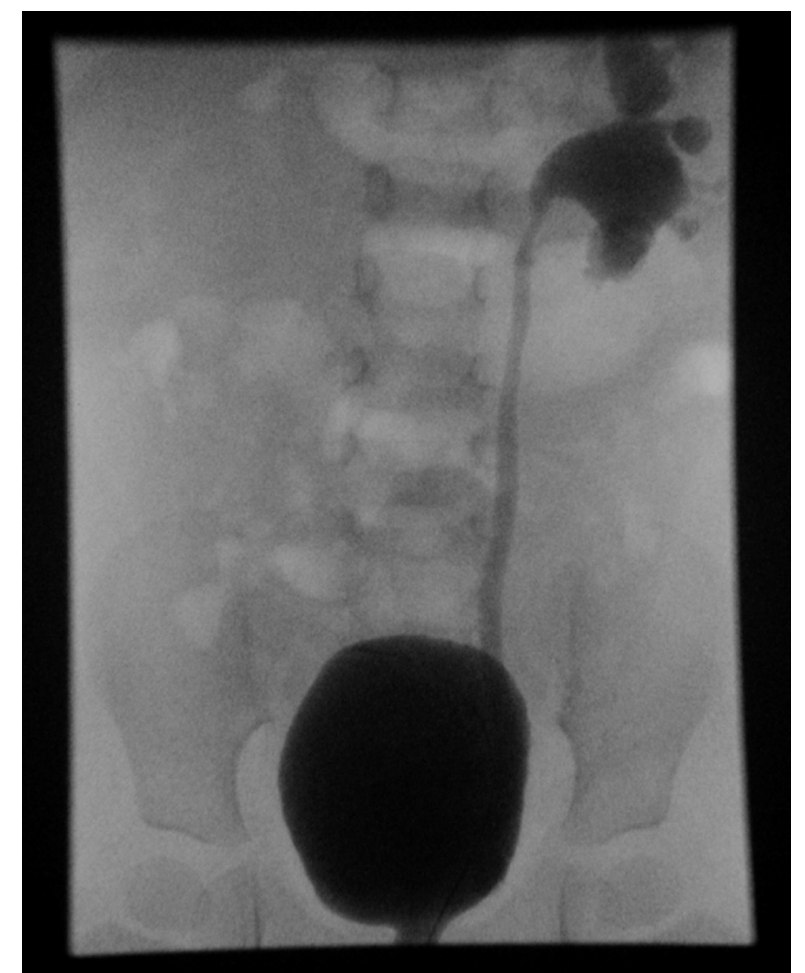

Figure $4 \mathrm{~A}$ voiding cystourethrogram of a patient with grade III reflux, characterized by mild ureteral dilatation and minimal blunting of the calyces.

\section{Screening for VUR in prenatal hydronephrosis}

Prenatal hydronephrosis (PNH) may represent a spectrum of urologic pathologies. VUR represents $15 \%-30 \%$ of cases of antenatal hydronephrosis. ${ }^{18}$ Consequently, postnatal evaluation affords the clinician the opportunity to diagnose and manage reflux before secondary injury is sustained due to urinary tract infection. While PNH is defined by the degree of anterior-posterior renal pelvic diameter, the magnitude of fetal renal pelvicaliectasis is not reliably predictive of reflux, and as of yet, there is currently no standard threshold of dilatation that can be used to predict which infants will benefit most from postnatal evaluation. ${ }^{19}$

RBUS is the first-line study for children with prenatally diagnosed hydronephrosis. Clinicians are urged to delay the first postnatal ultrasound until after the first few days of life, due to the relative dehydration and oliguria seen in the neonate. The absence of hydronephrosis on the first postnatal RBUS cannot reliably exclude reflux. However, the literature has shown that VUR is rare, or if present, likely low-grade, in infants with two normal successive postnatal ultrasounds. ${ }^{20}$ Additionally, studies have demonstrated that the degree of dilation does not correlate with VUR severity. ${ }^{21}$

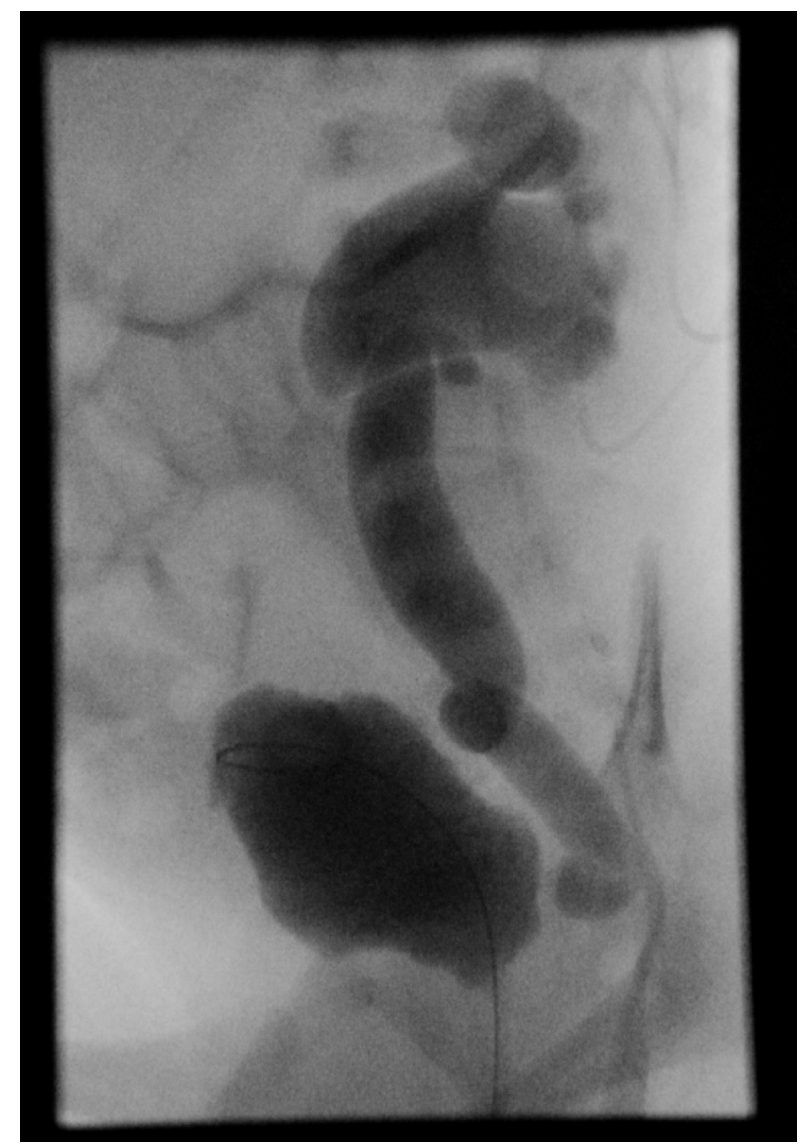

Figure 5 Grade $V$ vesicoureteral reflux, as demonstrated by marked ureteral tortuosity, pelvic dilatation, and severe calyceal blunting.

In the setting of PNH, the EAU advocates for the use of VCUG to screen for VUR in infants who present with bilateral hydronephrosis, bladder-wall thickening, renal cortical abnormalities, duplicated systems, and/or a ureterocele. Current AUA guidelines recommend proceeding with VCUG in cases of Society for Fetal Urology grade 3-4 hydronephrosis, abnormal bladder-wall findings, or in those who develop a UTI while on observation. ${ }^{15}$

\section{Screening for VUR in siblings}

VUR is a familial disorder, likely inherited in an autosomal dominant fashion, with increased prevalence in siblings and offspring of patients with VUR. Given the higher frequency of VUR in family members, much attention has been focused on developing screening protocols for asymptomatic siblings and offspring of reflux patients. The goal of screening in these individuals is to identify a high-risk population, and thus avert the complications of recurrent UTIs and renal scarring. However, some argue that screening an asymptomatic population results in unnecessary radiation exposure, parental distress, increased health-care costs, and overtreatment of 


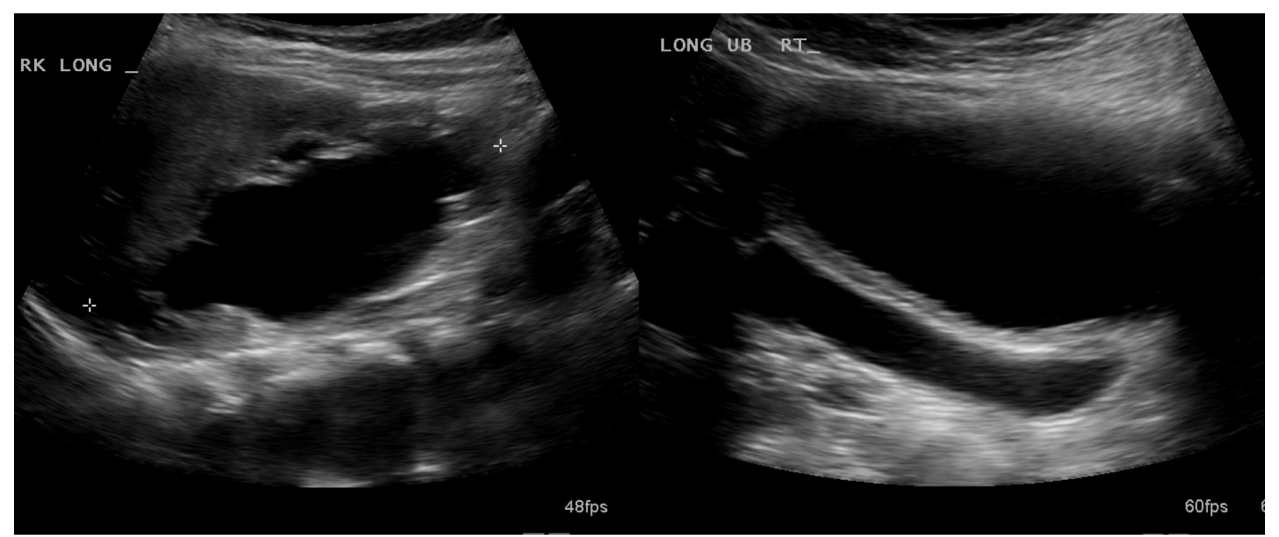

Figure 6 Ultrasound images in a patient with grade $V$ vesicoureteral reflux.

Note: Sonographic features of grade $\vee$ reflux may include marked ureteral dilation, moderate to severe hydronephrosis, and varying degrees of renal parenchymal thinning.

otherwise clinically insignificant disease. Unfortunately, there is a scarcity of high-level, randomized controlled data on screening asymptomatic siblings; therefore, current recommendations are based largely on expert opinion.

Menezes and Puri attempted to identify those VUR siblings who are most likely to be affected by clinically significant disease. In a prospective study of 215 families with VUR, they found the incidence of sibling reflux was maximal in patients younger than 3 years, VUR was high-grade in $85 \%$ of patients (including symptomatic and asymptomatic siblings), and renal scarring was present in $35 \%$ of siblings diagnosed after a UTI versus $15 \%$ among those who were screened. The authors conclude from their analysis that VUR screening would be most beneficial in all VUR siblings who are younger than 3 years of age. ${ }^{22}$ These findings are in keeping with several other studies that have shown a higher rate

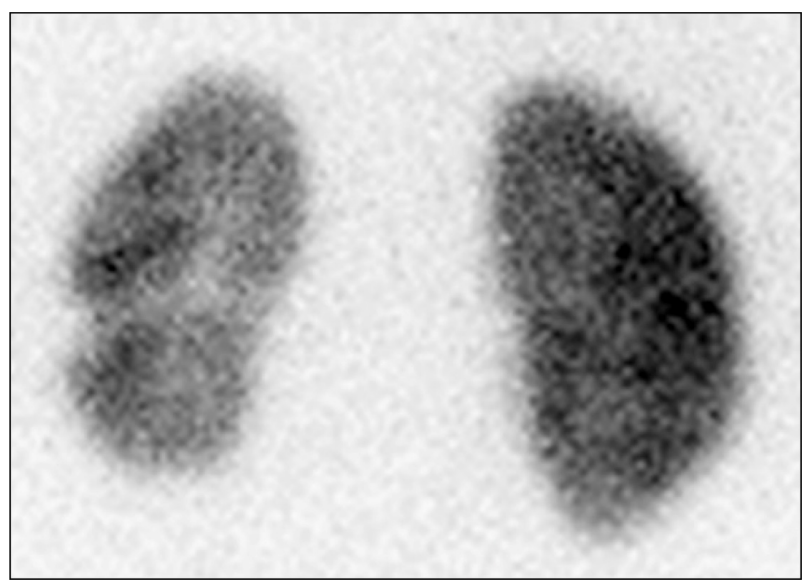

Figure 7 Dimercaptosuccinic acid scan in a young child 6 months after an episode of pyelonephritis demonstrates a focal, photopenic defect in the lower pole of the left kidney, indicative of renal scarring.

Note: Müller LSO. Imaging in urinary tract infection: top-down or down-up? Pediatr Radiol. 201 I;41:96-98. ${ }^{8}$ of reflux morbidity in first-, second-, and even third-degree relatives of index patients. Cascio et al also found that the incidence of renal injury was much higher (21\%) in siblings with VUR who presented with UTI than in asymptomatic siblings (2\%). ${ }^{23}$ Hunziker and Puri observed a $23 \%$ rate of renal scarring in first-degree relatives with VUR in addition to high rates of hypertension, end-stage renal disease, renal transplantation, and nephrectomy for reflux nephropathy in second- and third-degree relatives with VUR. ${ }^{24}$

The most recent EAU recommendations reflect the findings of these studies, recognizing the potential morbidity of unrecognized familial reflux, while also acknowledging the uncertain efficacy of antibiotic prophylaxis in preventing febrile UTIs. Their recommendations consequently question the value of widespread sibling screening. The EAU recommends informing parents of children with VUR of the increased familial prevalence, and offering early screening by means of RBUS. VCUG is only recommended in the presence of sonographic renal or bladder abnormalities, renal scarring, or history of prior UTI. Furthermore, screening in toilet-trained older VUR siblings is discouraged..$^{14}$ The AUA guidelines also emphasize incorporating parental preferences in sibling screening decisions, offering RBUS to non-toilet-trained VUR siblings, and only performing a VCUG in the presence of an imaging abnormality or prior UTI. ${ }^{4}$ Ultimately, sibling-screening recommendations are limited by a lack of high-quality literature, and thus are quite variable in practice and still largely influenced by physician opinion.

\section{Treatment options}

The goals of VUR treatment are to prevent recurrent febrile UTIs, avert renal injury, and minimize patient morbidity in 
terms of both treatment and follow-up. There are two main approaches to VUR management - conservative versus surgical. Conservative management approaches include watchful waiting, antibiotic prophylaxis, and bladder rehabilitation. Surgical treatment consists of endoscopic therapy, open surgical methods, and minimally invasive approaches (laparoscopic and robotic). The choice of treatment is complex and is driven by numerous factors, including patient age, sex, VUR grade, history of UTI, baseline renal function, physician and parental preference, and associated bowel/ bladder dysfunction.

\section{Conservative management}

The objective of conservative management is to prevent UTI while avoiding the potential harms of surgery. The underlying assumption in using this management approach is based on the understanding that VUR may resolve spontaneously in a large percentage of children. Resolution is approximately $85 \%$ in grade I, $75 \%$ in grade II, $50 \%-65 \%$ in grade III, $15 \%-35 \%$ in grade IV, and less than $10 \%$ in grade $V^{25,26}$ Unilateral reflux resolves more rapidly than bilateral disease, and boys tend to have higher rates of spontaneous resolution than girls. ${ }^{25,26} \mathrm{~A}$ watchful waiting approach may be employed in those with low-grade VUR and no prior history of urinary tract infection. Routine follow-up imaging with renal ultrasound and VCUG or nuclear cystography is performed to check for spontaneous resolution. At present, there is no consensus on the appropriate frequency of this follow-up system; however, biannual ultrasonography with annual cystography is a common approach. In cases of recurrent febrile infections, conservative management should be abandoned and an interventional approach considered.

Circumcision in the male infant with VUR may be considered due to the increased risk of UTI in this age group. A meta-analysis of twelve studies with over 402,000 children by Singh-Grewal et al found that circumcision is effective in reducing the risk of UTI. ${ }^{27}$ In uncircumcised male children with recurrent UTIs or high-grade reflux (grade III-V), the risk of UTI recurrence is $10 \%$ and $30 \%$ respectively, and the numbers needed to treat to prevent one UTI are eleven and four, compared to 111 in those without risk factors. ${ }^{27}$

\section{Antibiotic prophylaxis}

Perhaps no issue in VUR management has been as divisive as the role of continuous antibiotic prophylaxis (CAP). The literature on this topic is distributed between two extreme positions: the position that CAP clearly prevents UTI and renal injury versus the position that $\mathrm{CAP}$ has no benefit at all.
Potential harms of unnecessary antibiotic use include gastrointestinal side effects, increased antibiotic resistance, marrow suppression, and severe drug reactions. ${ }^{28}$ The most common agents employed are amoxicillin, trimethoprim, trimethoprim-sulfamethoxazole, and nitrofurantoin, all administered as a daily low-dose prescription.

The latest AUA guidelines $(2010)^{15}$ recommend the use of CAP in children under 1 year of age with VUR and a history of febrile UTI and in those children with grade III-V reflux without a history of infection. The EAU recommends CAP regardless of VUR grade, renal scarring or symptoms in all children diagnosed within the first year of life. ${ }^{14}$ The additional recommendation is made that CAP be continued until after toilet-training to protect children from UTI secondary to underlying lower urinary tract dysfunction (LUTD).

The role of CAP has been studied by many authors in order to understand better its overall efficacy, costeffectiveness, and to determine the most appropriate timing and duration. There are few studies, however, that have shown a significant reduction in recurrent UTIs with the use of antibiotic prophylaxis. As part of the multicenter, randomized, controlled Swedish reflux trial, Brandström et al found that girls on antibiotic therapy had a reduced rate of recurrent febrile UTI compared to those on surveillance alone (19\% versus 57\%). Interestingly, among boys there was no difference in rates of recurrent UTI between treatment arms. ${ }^{29}$ These results were limited to children with higher-grade (III-V) VUR, which may explain the observed positive treatment effect.

On the other side of the debate are those studies that have highlighted the lack of benefit of antibiotic prophylaxis. A multicenter, randomized, controlled trial of 100 Italian children with grades II-IV VUR found that CAP was ineffective for reducing the pyelonephritis recurrence rate and the incidence of renal scarring at a mean follow-up of 4 years. ${ }^{12}$ A larger US multicenter, randomized, controlled study by Garin et al additionally found no role for urinary antibiotic prophylaxis in preventing recurrent UTIs and the development of renal parenchymal damage in patients with mild/moderate VUR. ${ }^{30}$ Montini et al similarly found no decrease in the UTI recurrence rate in their randomized, controlled, open-label noninferiority trial comparing no prophylaxis with prophylaxis. ${ }^{13}$ Among these studies, the PRIVENT randomized trial of antibiotic prophylaxis was the only placebo-controlled, blinded study. The PRIVENT trial measured adherence rates on antibiotics throughout their study. Despite finding a very modest treatment effect in favor of antibiotics, the results were not statistically 
significant, and patient numbers were too small to adequately power the analysis. ${ }^{31}$ The recently updated Cochrane review (2011) of various interventions for VUR concluded that the small number of poor-quality studies collectively show no evidence in favor of the use of antibiotics to prevent recurrent UTI. ${ }^{32}$ An additional systematic review by Mori et al found no evidence for reduction of symptomatic UTI or incidence of renal scarring among those patients placed on antibiotic prophylaxis. ${ }^{33}$

One possible reason for the divergent results among studies of antibiotic prophylaxis is patient noncompliance on CAP. A recent analysis of a national pharmacy claims database by Copp et al found that antibiotic compliance was only around $40 \%$. Higher rates of adherence were seen in patients younger than 5 years of age, those who experienced frequent hospitalizations, and among those who were seen by a urologist on more than one occasion. ${ }^{34}$ Unfortunately, up to this point, many of the randomized studies evaluating the efficacy of CAP have only loosely accounted for patient compliance, thus complicating interpretation of much of the current data regarding antibiotic efficacy. Whether or not there are subgroups of VUR patients who would be best served by CAP still remains to be elucidated.

\section{Bladder/bowel rehabilitation}

It is well known that bowel dysfunction and LUTD play a critical role in VUR. LUTD has been posited as a contributor to the development of secondary VUR, as well as an important factor in the rate of UTI recurrence and spontaneous resolution of VUR. As part of the Swedish Reflux Trial in Children, a subgroup analysis of LUTD in terms of prevalence, VUR resolution rates, UTI recurrence, and development of renal damage was performed. ${ }^{35}$ The authors found that LUTD was seen in $34 \%$ of children with VUR, and recurrent urinary tract infections were found in $33 \%$ versus $20 \%$ of children with and without LUTD, respectively. This study also observed a lower rate of VUR resolution (22\% versus $56 \%$ ), and a much higher rate of renal damage (85\% versus $52 \%)$ among children with LUTD. Additionally, the authors noted a higher rate of grade IV reflux in those children with bladder dysfunction. The current AUA guidelines emphasize the need for a focused inquiry into the signs and symptoms of bowel and bladder dysfunction in the patient with VUR. Clinicians are urged to be particularly vigilant regarding these issues as the child moves through the phases of toilet training. ${ }^{15}$ Current options for bladder rehabilitation include bladder training with timed voiding, biofeedback, anticholinergic medications, alpha blockade, and constipation management.
It is critically important to address LUTD prior to proceeding to surgical therapy. Unfortunately, there are still very few data on VUR outcomes in patients who have undergone a program of bladder rehabilitation.

\section{Surgical therapy}

Surgical options for VUR management include endoscopic therapies, open surgical techniques, and minimally invasive forms of ureteral reimplantation. Current indications for antireflux surgery include recurrent or breakthrough febrile UTIs, high-grade VUR, VUR bilaterality, the development of renal scarring or functional compromise on scintigraphy, long-term persistence of VUR, concomitant urologic anomalies predisposing to persistent VUR (ie, paraureteral diverticula), and parental preference.

\section{Endoscopic therapy}

Endoscopic injection of periureteral bulking agents has been used for the management of VUR since the $1980 \mathrm{~s} .{ }^{36}$ The procedure is performed cystoscopically by a submucosal injection of a biocompatible bulking agent beneath the intravesical portion of the ureter. It is hypothesized that the bulking agent thus elevates and narrows the distal ureter and ureteral orifice, preventing retrograde passage of urine while still allowing antegrade flow. A variety of agents have been employed over the years, including polytetrafluoroethylene (Teflon ${ }^{\circledR}$; DuPont Co, West Chester, PA, USA), collagen, autologous fat, silicone, chondrocytes, and most commonly dextranomer/ hyaluronic acid (Dx/HA or Deflux ${ }^{\circledR}$; Salix Pharmaceuticals, Raleigh, NC, USA). Many of these agents have not withstood the test of time, and currently Deflux is the most studied and widely used bulking agent. The use of Deflux has increased exponentially in the past several years. Lendvay et al performed a patient care database study among 37 participating hospitals in order to evaluate national trends in VUR treatment, finding a $288 \%$ increase in the utilization of endoscopic therapy between 2002 and 2004. ${ }^{37}$ By comparison, the rate of open reflux procedures remained unchanged, and they noted a shifting balance in reflux management from open to endoscopic therapy in more than $60 \%$ of enrolled institutions.

Success rates of Deflux injection for VUR vary widely across the published literature. Additionally, clinical validation of endoscopic injection therapy has been limited by low reporting quality, marked study population heterogeneity, variable definitions of treatment success, small sample size, and limited patient follow-up. In a systematic review of over 47 studies by Routh et al, they found an overall 77\% success rate of a single application of $\mathrm{Dx} / \mathrm{HA}$ at 3 months, noting 
however that individually reported success rates were widely divergent across the included studies. ${ }^{38}$ In a meta-analysis of over 5527 patients, the reflux resolution rate after a single endoscopic treatment was 78\% for grades I and II, 72\% for grade III, $63 \%$ for grade IV, and $51 \%$ for grade V. Therapeutic success was reduced in duplex collecting system VUR (50\%) and in those with a neuropathic bladder (62\%) compared to those with single collecting systems (73\%) and normal bladders (74\%). ${ }^{39}$ The cumulative success rate increased with successive treatments, to a maximum of $85 \%$, which is still lower than the reported $\sim 95 \%$ success rate of open surgery. The three treatment arms of the Swedish reflux trial, which included 203 children with grade III-IV VUR randomized to endoscopic injection, CAP, or surveillance alone, reported that endoscopic treatment resulted in the highest rate of VUR resolution, 71\%, compared with the other treatment arms (CAP 39\% and surveillance 48\%). There was no difference in rates of febrile UTIs between the prophylaxis and endoscopic therapy groups. Finally, the reported recurrence rate after endoscopic treatment was $20 \%$ at the 2-year follow-up interval. ${ }^{40}$ Unfortunately, as of yet, there have been no randomized clinical trials that have compared endoscopic correction of VUR with open ureteral reimplantation.

The significant costs of endoscopic therapy for VUR have also been examined. The cost-efficacy of Dx/HA compared to open surgical correction was queried by Saperston et al, who found that the average total system reimbursement for a single endoscopic treatment of unilateral reflux was estimated at US\$4259 per patient compared to \$3945 for open extravesical ureteral reimplantation (calculating a $3 \%$ hospital admission rate). ${ }^{41}$ The cost of a single vial of Dx/HA was most recently valued at $\$ 1900$, thus accounting for much of the procedural cost. ${ }^{42}$

Despite the limited literature on the topic, endoscopic VUR therapy remains a reasonable alternative for low-grade reflux, as it offers a safe, minimally invasive, moderately effective outpatient surgical treatment for children with VUR. However, parents should be adequately counseled about the inadequate long-term data on its durability and the potential need for repeat intervention.

\section{Open surgery}

A variety of intravesical and extravesical approaches to ureteral reimplantation (ureteroneocystostomy) have been described for the surgical correction of VUR. Open surgical methods include the Cohen cross-trigonal advancement, ${ }^{43}$ Politano-Leadbetter suprahiatal approach, ${ }^{44}$ Glenn-Anderson infrahiatal reimplantation, ${ }^{45}$ and the
Lich-Gregoir extravesical ureteroneocystostomy. Each method has its own distinct advantages and complications, but with an overall success rate of $95 \%-98 \%{ }^{46}$ The most commonly employed open method is Cohen cross-trigonal ureteral reimplantation. Independent of technique, all open operations share the basic goal of lengthening the submucosal tunnel to provide a 5:1 tunnel length-to-diameter ratio while avoiding denuding or kinking the ureter, which can lead to devascularization and obstruction. Surgical complications include bleeding, infection, ureteral obstruction, and persistent ipsilateral or new contralateral reflux. Notably, bilateral extravesical reimplantation carries an increased risk of postoperative urinary retention. While the techniques have largely remained unchanged, several improvements in patient care, including the minimization of incision size, shorter operative stays, and decreased catheter use, have made the open approach a less morbid intervention.

Unfortunately, there are very few studies that have compared clinical outcomes of medical versus open surgical therapy for VUR management. This is largely due to the fact that randomization of children to surgical interventions is fraught with ethical, social, and methodological difficulties. ${ }^{47}$ However, recent data from the International Reflux Study in Children provides some evidence that surgical management offers little advantage in the clinical outcomes that matter. This multicenter international trial of children with grades III/IV VUR randomized to either medical or open surgical treatment found no difference in UTI recurrence rate, renal growth or function, or incidence of new renal scarring at 10 years of follow-up. ${ }^{48}$ These results echo prior findings from a small randomized trial of medical versus surgical intervention published by Smellie et al, who found no improvement in renal outcomes among those children with bilateral, severe VUR, and preexisting reflux nephropathy that underwent surgical correction. ${ }^{49}$ These studies highlight that neither medical nor open surgical therapy can completely protect children from renal damage, and efforts must continue to identify more rapidly at-risk patients before the onset of renal scarring and nephropathy.

\section{Minimally invasive approaches}

Recent advancements have been made in both laparoscopic and robotic approaches to ureteral reimplantation. Laparoscopic extravesical ureteral reimplantation has gained acceptance as a safe and effective treatment associated with minimal morbidity. In a single-institution retrospective analysis, Bayne et al reported a success rate $(95 \%)$ for the laparoscopic extravesical approach that was 
equivalent to the gold-standard open approach, even in children with complex ureteral anatomy. ${ }^{50}$ Canon et al reported their preliminary outcomes with vesicoscopic cross-trigonal ureteral reimplantation, noting the procedure involved less pain than open transvesical surgery and yet featured comparable VUR resolution rates (91\% versus 97\%). ${ }^{51}$ Unfortunately, widespread use of pediatric laparoscopy has been limited by the steep learning curve, most notably related to the demands of intracorporeal suturing and knottying within the very small abdominopelvic space of the pediatric patient. The longer operative times reported for laparoscopy have additionally prevented it from supplanting the open approach.

Robotic-assisted ureteral reimplantation, with its improved three-dimensional optics, 10× magnification and enhanced manual dexterity, eliminates many of the difficulties of conventional pediatric laparoscopy. Early experience with robotic reimplantation was noted for its lower success rates and higher frequency of postoperative complications. Peters was the first to describe the robotic pediatric ureteroneocystostomy in 2004, with a VUR correction rate of $89 \%$ and a $12 \%$ complication rate. ${ }^{52}$ Lendvay had a lower success rate in a preliminary series of robotic extravesical reimplants, $75 \%$, and a similarly high complication rate of $12 \% .{ }^{53}$ The most recent robotic literature, however, has shown equivalent success rates with reduced patient morbidity. Kasturi et al reported a $99 \%$ VUR resolution rate with no postoperative voiding dysfunction in their series of 150 patients who underwent bilateral nerve-sparing extravesical robotic-assisted laparoscopic ureteral reimplantation. ${ }^{54}$ Marchini et al recently reported their outcomes with both intravesical and extravesical robotic-assisted laparoscopic ureteral reimplantation. ${ }^{55}$ Compared to case-matched controls consisting of patients who underwent intravesical or extravesical open ureteral reimplantation, there was no difference in either clinically or radiologically defined success, and the robotic approach was associated with reduced bladder spasm, hematuria, catheter duration, and hospital stays. However, in the early phase of this series, the various complications of the robotic approach, ie, postoperative urinary retention and bladder and ureteral leak, led to a higher rate of reintervention.

To date, the application of robotics to pediatric surgery has been limited by increased hospital costs, longer operative times, and the need for special training of ancillary staff. Ultimately, further studies are also necessary to define the costs and benefits of these minimally invasive approaches relative to the gold standard of open surgery prior to their wholesale adoption.

\section{VUR cost-effectiveness analysis}

Recognizing that determining the ideal treatment algorithm for VUR is fraught with difficulty due to poor-quality data, Hsieh et $\mathrm{al}^{56}$ performed a cost-effectiveness analysis using Markov models of five different treatment protocols for boys and girls with moderate-grade VUR. By assigning higher utility values to those protocols that minimized treatment and disease burden, they determined that a noninterventional approach represented the least costly and highest-utility therapy. Increasingly sophisticated decision analyses are likely on the horizon, as data from additional clinical trials comparing the various treatment methods for VUR become available for analysis.

\section{Parental preferences}

Given the flawed literature, the lack of overall consensus on management, and the scant absolute indications for surgical intervention for VUR, clinicians have incorporated parental preference as an important factor in clinical decisionmaking. Studies by Ogan et $\mathrm{al}^{57}$ and Krill et $\mathrm{al}^{58}$ found that parents of children with VUR generally preferred antibiotic prophylaxis as the initial treatment modality. When faced with the need for continued treatment beyond 3-4 years, the majority of parents preferred endoscopic treatment to open surgery, despite being informed of the lower success rate. These findings contrast with those previously described by Capozza et al, who found an overwhelming preference for endoscopic surgery $(80 \%)$ rather than antibiotic prophylaxis $(5 \%)$ or open surgery $(2 \%) .{ }^{59} \mathrm{~A}$ more recent study by Hsieh et $\mathrm{al}^{60}$ described their results of a cross-sectional analysis of how parents elect to have VUR managed in their children, with a particular focus on the influence of racial, ethnic, and socioeconomic factors. Interestingly, they found that parents valued the opinion of their pediatric urologist above all else, and that the financial burden of VUR management was a stronger factor in decision-making among Hispanic families. These studies highlight the need to further elucidate parental decision-making factors and incorporate parental preferences in care of children with VUR.

\section{Follow-up recommendations}

The AUA and EAU recommend ongoing monitoring of all children with VUR until the reflux has resolved or is no longer significant. Long-term follow-up protocols are intended to identify urinary tract infections and early signs of renal insufficiency. There is no gold-standard approach; however, most guidelines agree on periodic imaging and urinalysis. A commonly employed algorithm includes annual urinalysis 
to screen for infection and proteinuria. Routine urine cultures are not recommended unless there are signs of infection. Interval imaging, every 6-12 months, with renal ultrasonography and VCUG is important to monitor for spontaneous resolution or renal deterioration.

\section{Uncertainties and future directions}

VUR has become an exciting area for clinical, basic science, and translational research. Currently, several innovative imaging modalities are on the horizon. Improvements in ultrasonographic contrast agents (using stabilized air-filled microbubbles) along with enhanced tissue and contrastspecific harmonic imaging have led to higher sensitivity and specificity in the detection and grading of VUR with voiding urosonography. ${ }^{61}$ Magnetic resonance voiding cystograms are potential additions to the imaging armamentarium, providing enhanced anatomic detail while eliminating ionizing radiation exposure to the pediatric patient. However, magnetic resonance imaging remains a very costly study and requires both invasive catheterization and sedation, thus leaving its added value a real question. ${ }^{9}$ Noninvasive, nonionizing imaging facilitated by transabdominal manipulation of bladder temperature is still in the early stages of development, but holds hope for future clinical application in the coming years. ${ }^{62}$

We can also look forward to the much-anticipated findings from the Randomized Intervention for Children with Vesicoureteral Reflux study, a multicenter, double-blind, randomized, placebo-controlled trial with over 15 participating institutions and more than 600 enrolled pediatric patients. The study is designed to assess UTI recurrence, renal scarring, antimicrobial resistance, patient quality of life, medication compliance, resource utilization, and VUR status in children randomized to antibiotic prophylaxis versus placebo. ${ }^{63}$

Finally, ongoing basic science research in biomarker discovery (serum basic fibroblast growth factor, ${ }^{64}$ procalcitonin, ${ }^{65,66}$ and urine interleukin 8$)^{67}$ and urinary proteomics are being directed towards the development of new, noninvasive tests to diagnose and perhaps prognosticate children with VUR. ${ }^{68}$

\section{Conclusion}

The available literature regarding VUR screening, imaging, and treatment continues to grow, resolving some areas of controversy while leaving new questions to be answered. Despite the heterogeneous quality of the published literature, there are multiple areas of consensus on the basic principles of management to help guide the clinician in charge of the pediatric reflux patient. We can expect VUR management to continue to evolve as new imaging options are explored, further randomized controlled trials are undertaken, improved risk-stratification models are developed, and biomarker research is translated into clinical practice.

\section{Disclosure}

The authors report no conflicts of interest in this work.

\section{References}

1. Bailey R. Vesicoureteral reflux in healthy infants and children. In: Hodson J, Kincaid-Smith P, editors. Reflux Nephropathy. New York: Masson; 1979:59-61.

2. Sargent MA. What is the normal prevalence of vesicoureteral reflux? Pediatr Radiol. 2000;30:587-593.

3. Chand DH, Rhoades T, Poe SA, Kraus S, Strife CF. Incidence and severity of vesicoureteral reflux in children related to age, gender, race and diagnosis. $J$ Urol. 2003;170:1548-1550.

4. Skoog SJ, Peters CA, Arant BS Jr, et al. Pediatric Vesicoureteral Reflux Guidelines Panel summary report: clinical practice guidelines for screening siblings of children with vesicoureteral reflux and neonates/ infants with prenatal hydronephrosis. J Urol. 2010;184:1145-1151.

5. Lebowitz RL, Olbing H, Parkkulainen KV, Smellie JM, TamminenMöbius TE. International system of radiographic grading of vesicoureteric reflux. International Reflux Study in Children. Pediatr Radiol. 1985;15:105-109.

6. Estrada CR Jr, Passerotti CC, Graham DA, et al. Nomograms for predicting annual resolution rate of primary vesicoureteral reflux: results from 2,462 children. $J$ Urol. 2009;182:1535-1541.

7. Piscitelli A, Galiano R, Serrao F, et al. Which cystography in the diagnosis and grading of vesicoureteral reflux? Pediatr Nephrol. 2007;23:107-110.

8. Müller LSO. Imaging in urinary tract infection: top-down or down-up? Pediatr Radiol. 2011;41:96-98.

9. Snow BW. New noninvasive methods to diagnose vesicoureteral reflux. Curr Opin Urol. 2011;21:339-342.

10. [No authors listed]. Practice parameter: the diagnosis, treatment, and evaluation of the initial urinary tract infection in febrile infants and young children. American Academy of Pediatrics. Committee on Quality Improvement. Subcommittee on Urinary Tract Infection. Pediatrics. 1999;103:843-852.

11. Roberts KB. Urinary tract infection: clinical practice guideline for the diagnosis and management of the initial UTI in febrile infants and children 2 to 24 months. Pediatrics. 2011;128:595-610.

12. Pennesi M, Travan L, Peratoner L, et al. Is antibiotic prophylaxis in children with vesicoureteral reflux effective in preventing pyelonephritis and renal scars? A randomized, controlled trial. Pediatrics. 2008;121:e1489-e1494.

13. Montini G, Rigon L, Zucchetta P, et al. Prophylaxis after first febrile urinary tract infection in children? A multicenter, randomized, controlled, noninferiority trial. Pediatrics. 2008;122:1064-1071.

14. Tekgül S, Riedmiller H, Hoebeke P, et al. EAU guidelines on vesicoureteral reflux in children. Eur Urol. 2012;62:534-542.

15. Peters CA, Skoog SJ, Arant BS Jr, et al. Summary of the AUA guideline on management of primary vesicoureteral reflux in children. $J$ Urol. 2010;184:1134-1144

16. National Collaborating Centre for Women's and Children's Health (UK). Urinary Tract Infection in Children: Diagnosis, Treatment and Long-Term Management. London: RCOG Press; 2007.

17. Herz D, Merguerian P, McQuiston L, Danielson C, Gheen M, Brenfleck L. 5-Year prospective results of dimercapto-succinic acid imaging in children with febrile urinary tract infection: proof that the top-down approach works. J Urol. 2010;184:1703-1709. 
18. Gokce I, Biyikli N, Tugtepe H, Tarcan T, Alpay H. Clinical spectrum of antenatally detected urinary tract abnormalities with respect to hydronephrosis at postnatal ultrasound scan. Pediatr Surg Int. 2012;28:543-552.

19. Coplen DE, Austin PF, Yan Y, Dicke JM. Correlation of prenatal and postnatal ultrasound findings with the incidence of vesicoureteral reflux in children with fetal renal pelvic dilatation. J Urol. 2008;180: 1631-1634; discussion 1634.

20. Hafez AT, McLorie G, Bagli D, Khoury A. Analysis of trends on serial ultrasound for high grade neonatal hydronephrosis. $J$ Urol. 2002;168:1518-1521.

21. Nguyen HT, Herndon CD, Cooper C, et al. The Society for Fetal Urology consensus statement on the evaluation and management of antenatal hydronephrosis. J Pediatr Urol. 2010;6:212-231.

22. Menezes M, Puri P. Familial vesicoureteral reflux - is screening beneficial? J Urol. 2009;182:1673-1677.

23. Cascio S, Yoneda A, Chertin B, Colhoun E, Puri P. Renal parenchymal damage in sibling vesicoureteric reflux. Acta Paediatr. 2003;92:17-20.

24. Hunziker M, Puri P. Familial vesicoureteral reflux and reflux related morbidity in relatives of index patients with high grade vesicoureteral reflux. $J$ Urol. 2012;188:1463-1466.

25. Schwab CW Jr, Wu HY, Selman H, Smith GH, Snyder HM 3rd, Canning DA. Spontaneous resolution of vesicoureteral reflux: a 15-year perspective. J Urol. 2002;168:2594-2599.

26. Zerati Filho M, Calado AA, Barroso U Jr, Amaro JL. Spontaneous resolution rates of vesicoureteral reflux in Brazilian children: a 30-year experience. Int Braz J Urol. 2007;33:204-212; discussion 213-215.

27. Singh-Grewal D, Macdessi J, Craig J. Circumcision for the prevention of urinary tract infection in boys: a systematic review of randomised trials and observational studies. Arch Dis Child. 2005;90:853-858.

28. Elder JS, Peters CA, Arant BS Jr, et al. Pediatric Vesicoureteral Reflux Guidelines Panel summary report on the management of primary vesicoureteral reflux in children. J Urol. 1997;157:1846-1851.

29. Brandström P, Esbjörner E, Herthelius M, Swerkersson S, Jodal U, Hansson S. The Swedish reflux trial in children: III. Urinary tract infection pattern. J Urol. 2010;184:286-291.

30. Garin EH, Olavarria F, Garcia Nieto V, Valenciano B, Campos A, Young L. Clinical significance of primary vesicoureteral reflux and urinary antibiotic prophylaxis after acute pyelonephritis: a multicenter, randomized, controlled study. Pediatrics. 2006;117:626-632.

31. Craig JC, Simpson JM, Williams GJ, et al. Antibiotic prophylaxis and recurrent urinary tract infection in children. $N$ Engl $J$ Med. 2009;361:1748-1759.

32. Nagler EV, Williams G, Hodson EM, Craig JC. Interventions for primary vesicoureteric reflux. Cochrane Database Syst Rev. 2011;6:CD001532.

33. Mori R, Fitzgerald A, Williams C, Tullus K, Verrier-Jones K, Lakhanpaul M. Antibiotic prophylaxis for children at risk of developing urinary tract infection: a systematic review. Acta Paediatr. 2009;98:1781-1786.

34. Copp HL, Nelson CP, Shortliffe LD, et al. Compliance with antibiotic prophylaxis in children with vesicoureteral reflux: results from a national pharmacy claims database. J Urol. 2010;183:1994-1999.

35. Sillén U, Brandström P, Jodal U, et al. The Swedish reflux trial in children: v. Bladder dysfunction. J Urol. 2010;184:298-304.

36. O'Donnell B, Puri P. Treatment of vesicoureteric reflux by endoscopic injection of Teflon. Br Med J (Clin Res Ed). 1984;289:7-9.

37. Lendvay TS, Sorensen M, Cowan CA, Joyner BD, Mitchell MM, Grady RW. The evolution of vesicoureteral reflux management in the era of dextranomer/hyaluronic acid copolymer: a pediatric health information system database study. J Urol. 2006;176:1864-1867.

38. Routh JC, Inman BA, Reinberg Y. Dextranomer/hyaluronic acid for pediatric vesicoureteral reflux: systematic review. Pediatrics. 2010;125:1010-1019.

39. Elder JS, Diaz M, Caldamone AA, et al. Endoscopic therapy for vesicoureteral reflux: a meta-analysis. I. Reflux resolution and urinary tract infection. J Urol. 2006;175:716-722.
40. Holmdahl G, Brandström P, Läckgren G, et al. The Swedish reflux trial in children: II. Vesicoureteral reflux outcome. J Urol. 2010;184:280-285.

41. Saperston K, Smith J, Putman S, et al. Endoscopic subureteral injection is not less expensive than outpatient open reimplantation for unilateral vesicoureteral reflux. $J$ Urol. 2008;180:1626-1629; discussion 1629-1630.

42. Diamond DA, Mattoo TK. Endoscopic treatment of primary vesicoureteral reflux. N Engl J Med. 2012;366:1218-1226.

43. Cohen SJ. The Cohen reimplantation technique. Birth Defects Orig Artic Ser. 1977;13:391-395.

44. Politano VA, Leadbetter WF. An operative technique for the correction of vesicoureteral reflux. $J$ Urol. 1958;79:932-941.

45. Glenn JF, Anderson EE. Distal tunnel ureteral reimplantation. J Urol. 1967;97:623-626.

46. Duckett JW, Walker RD, Weiss R. Surgical results: International Reflux Study in Children - United States branch. J Urol. 1992;148: $1674-1675$.

47. [No authors listed]. Prospective trial of operative versus non-operative treatment of severe vesicoureteric reflux in children: five years' observation. Birmingham Reflux Study Group. Br Med J (Clin Res Ed). 1987;295:237-241.

48. Jodal U, Smellie JM, Lax H, Hoyer PF. Ten-year results of randomized treatment of children with severe vesicoureteral reflux. Final report of the International Reflux Study in Children. Pediatr Nephrol. 2006;21:785-792.

49. Smellie JM, Barratt TM, Chantler C, et al. Medical versus surgical treatment in children with severe bilateral vesicoureteric reflux and bilateral nephropathy: a randomised trial. Lancet. 2001;357:1329-1333.

50. Bayne AP, Shoss JM, Starke NR, Cisek LJ. Single-center experience with pediatric laparoscopic extravesical reimplantation: safe and effective in simple and complex anatomy. J Laparoendosc Adv Surg Tech A. 2012;22:102-106.

51. Canon SJ, Jayanthi VR, Patel AS. Vesicoscopic cross-trigonal ureteral reimplantation: a minimally invasive option for repair of vesicoureteral reflux. J Urol. 2007;178:269-273; discussion 273.

52. Peters CA. Robotically assisted surgery in pediatric urology. Urol Clin North Am. 2004;31:743-752.

53. Lendvay T. Robotic-assisted laparoscopic management of vesicoureteral reflux. Adv Urol. 2008:732942.

54. Kasturi S, Sehgal SS, Christman MS, Lambert SM, Casale P. Prospective long-term analysis of nerve-sparing extravesical robotic-assisted laparoscopic ureteral reimplantation. Urology. 2012;79:680-683.

55. Marchini GS, Hong YK, Minnillo BJ, et al. Robotic assisted laparoscopic ureteral reimplantation in children: case matched comparative study with open surgical approach. J Urol. 2011;185:1870-1875.

56. Hsieh MH, Swana HS, Baskin LS, Meng MV. Cost-utility analysis of treatment algorithms for moderate grade vesicoureteral reflux using markov models. J Urol. 2007;177:703-709.

57. Ogan K, Pohl HG, Carlson D, Belman AB, Rushton HG. Parental preferences in the management of vesicoureteral reflux. $J$ Urol. 2001;166:240-243.

58. Krill AJ, Pohl HG, Belman AB, Skoog SJ, Snodgrass WT, Rushton HG. Parental preferences in the management of vesicoureteral reflux. J Urol. 2011;186:2040-2044.

59. Capozza N, Lais A, Matarazzo E, Nappo S, Patricolo M, Caione P. Treatment of vesico-ureteric reflux: a new algorithm based on parental preference. BJU Int. 2003;92:285-288.

60. Hsieh MH, Madden-Fuentes RJ, Bayne A, et al. Cross-sectional evaluation of parental decision making factors for vesicoureteral reflux management in children. $J$ Urol. 2010;184(4 Suppl):1589-1593.

61. Zimbaro G, Ascenti G, Visalli C, et al. Contrast-enhanced ultrasonography (voiding urosonography) of vesicoureteral reflux: state of the art. Radiol Med. 2007;112:1211-1224.

62. Arunachalam K, Maccarini PF, De Luca V, Bardati F, Snow BW, Stauffer PR. Modeling the detectability of vesicoureteral reflux using microwave radiometry. Phys Med Biol. 2010;55:5417-5435. 
63. Keren R, Carpenter MA, Hoberman A, et al. Rationale and design issues of the Randomized Intervention for Children With Vesicoureteral Reflux (RIVUR) study. Pediatrics. 2008;122 Suppl 5:S240-S250.

64. Kobayashi H, Miyakita H, Yamataka A, Koga H, Lane GJ, Miyano T. Serum basic fibroblast growth factor as a marker of reflux nephropathy. J Pediatr Surg. 2004;39:1853-1855.

65. Leroy S, Adamsbaum C, Marc E, et al. Procalcitonin as a predictor of vesicoureteral reflux in children with a first febrile urinary tract infection. Pediatrics. 2005;115:e706-e709.

66. Mantadakis E, Plessa E, Vouloumanou EK, Karageorgopoulos DE, Chatzimichael A, Falagas ME. Serum procalcitonin for prediction of renal parenchymal involvement in children with urinary tract infections: a meta-analysis of prospective clinical studies. J Pediatr. 2009; 155:875-881. e1.
67. Galanakis E, Bitsori M, Dimitriou H, Giannakopoulou C, Karkavitsas NS, Kalmanti M. Urine interleukin- 8 as a marker of vesicoureteral reflux in infants. Pediatrics. 2006;117:e863-e867.

68. Drube J, Schiffer E, Lau E, et al. Urinary proteome analysis to exclude severe vesicoureteral reflux. Pediatrics. 2012;129:e356-e363.

69. International Reflux Committee. Medical versus surgical treatment of primary vesicoureteral reflux. Pediatrics. 1981;67:392.

\section{Publish your work in this journal}

Pediatric Health, Medicine and Therapeutics is an international, peerreviewed, open access journal publishing original research, reports, editorials, reviews and commentaries. All aspects of health maintenance, preventative measures and disease treatment interventions are addressed within the journal. Practitioners from all disciplines are invited to submit their work as well as healthcare researchers and patient support groups. The manuscript management system is completely online and includes a very quick and fair peer-review system. Visit http://www.dovepress.com/ testimonials.php to read real quotes from published authors.

Submit your manuscript here: http://www.dovepress.com/pediatric-health-medicine-and-therapeutics-journal 